\title{
Biceps Tenodesis is a Viable Option for Management of Proximal Biceps Injuries in Patients Less Than 25 Years of Age
}

Justin W. Griffin, MD1, Gregory L. Cvetanovich, MD¹, Jonathan Charles Riboh, MD², Timothy Sean Leroux, MD, MEd¹, Bernard R. Bach, MD¹, Gregory P. Nicholson, MD¹, Brian J. Cole, MD, MBA ${ }^{1}$, Nikhil N. Verma, MD³, Anthony A. Romeo, $\mathrm{MD}^{3}$

${ }^{1}$ Rush University Medical Center, Chicago, IL, USA, ${ }^{2}$ Duke Sports Science Institute, Durham, NC, USA, ${ }^{3}$ Midwest Orthopaedics at Rush, Chicago, IL, USA.

Objectives: Biceps tenodesis has been proposed as an alternative to superior labral anterior posterior (SLAP) repair in older individuals, and there is evidence to suggest it is a reliable operation with low failure rate in these patients. Despite this, there has been some trepidation to adopt this in young active patients due to concern for altered postoperative glenohumeral kinematics and stability. To date no studies have examined younger individuals less than 25 undergoing proximal biceps tenodesis as an intervention for proximal biceps related pain and dysfunction.

Methods: Between February 2011 and February 2013, 45 consecutive patients underwent biceps tenodesis for a diagnosis of biceps tendinopathy or biceps-labral complex injuries including SLAP tears and those with at least two-year follow up were analyzed. Thirty-five patients (77\%), with a mean age of $19.8 \pm 2.5$ years were available for follow-up at a mean of $38.6 \pm 9.5$ months postoperatively. Functional outcomes were assessed with Visual Analog Scale (VAS), American Shoulder and Elbow Surgeons (ASES) Score, ASES Functional Score, Simple Shoulder Test (SST), and range of motion (ROM). Patient activity level and return to sport was followed postoperatively. Pre and postoperative scores were compared utilizing paired t-tests between groups.

Results: 35 patients less than 25 years of age underwent biceps tenodesis and were available for follow up at two years (77\%). 33 of 35 patients were athletes with 15 patients playing at the collegiate level with softball and baseball representing the most common sports. 22 patients were overhead athletes. 16 patients had no prior shoulder surgery with 19 representing revision shoulder procedures. Overall, mean VAS improved from $5.0 \pm 1.2$ to $2.0 \pm 1.1$ ( $p<0.0001$ ). All clinical outcome scores improved with ASES score improving from 54.7 to 81.7 (mean difference 27.0 $\pm 3.7,95 \% \mathrm{Cl} 19.5,34.6)$, ( $p<0.0001$ ), ASES functional score improved from 17.5 to 25.1 (7.5 \pm 1.2 , $95 \% \mathrm{Cl} 5.0,10.0)(p<0.001)$, and SST improved from 7.4 to $10.1(2.7 \pm 0.5,95 \% \mathrm{Cl} 1.7,3.7)(p<0.0001)$. Range of motion was maintained postoperatively. Patients with higher BMI trended toward less improvement in function $(p=0.0854)$ and SST $(p=0.0355)$. At the time of follow-up, four patients (11\%) had undergone revision surgery. 22 of 33 (66\%) patients returned to sports with 16 returning at the same level and 6 at a lower level of play. 16 of 21 (76\%) overhead athletes return to sport with 4 requiring additional surgery after biceps tenodesis. There was no difference in scores for overhead versus nonoverhead athletes.

Conclusion: In the present study, biceps tenodesis in patients less than 25 years of age yields excellent outcomes with two thirds of patients able to return to sport. Additionally, we found a low revision rate. Of note, BMI appears to be a risk factor for a negative outcome following biceps tenodesis. Future long-term multicenter comparative studies with long term follow up are needed to demonstrate durability of biceps tenodesis in young patients.

The Orthopaedic Journal of Sports Medicine, 5(3)(suppl 3)

DOI: $10.1177 / 2325967117$ S00116

(C)The Author(s) 2017

This open-access article is published and distributed under the Creative Commons Attribution - NonCommercial - No Derivatives License (http://creativecommons.org/licenses/by-nc-nd/3.0/), which permits the noncommercial use, distribution, and reproduction of the article in any medium, provided the original author and source are credited. You may not alter, transform, or build upon this article without the permission of the Author(s). For reprints and permission queries, please visit SAGE's Web site at http://www.sagepub.com/journalsPermissions.nav. 